\title{
MANAGEMENT OF THE BUILDING SITE: SENSORS NETWORK AND
}

\section{BIM}

\author{
Mauro De Luca Picione \\ School of Engineering (SI) University of the Basilicata, m.delucapicione@gmail.com
}

Keywords: BIM, Building Site, Safety, Planning, Checks.

\section{INTRODUCTION}

The sector of the constructions continues to be a sector with high-level of dangerousness for the employees, with high percentages of accidents and significant incidences of professional illnesses. The reports of the syndical associations and category confirm that the yard continues to be a place with an intrinsic dangerousness. Insofar the belief that safety cannot be considered an option of the productive trial is surely the aim of the sector of the constructions. Safety on the job is a qualifying element of a productive trial that must be projected, on purpose, for every working phase only after a careful evaluation of the risks that, nevertheless will not be not enough if not matched with a constant control, in case redefined during exercise.

It's known that to face the theme of the safety in the building yards It is necessary to recognize the peculiar characters of the productive reality or the relationship among the varying spacetime that don't have comparison with other productive sectors, but above all the relationship of the productive activities with the spaces and the environment. The relationship with the site with respect to the advancement of the jobs often drives to determine danger conditions due to improvisation or toanticipated operational choices. These factors cause the building site to be a place of job that exposes the employees to particularly elevated risks, with an high rate of mortality as it is drawn by I.N.A.I.L. statistic data. As it is well shared, the introduction of safety elements in the building sites is not an easy activity since it has an intrinsic difficulty to pick up management and control data. Therefore the organization of the building yard needs an intense job of planning, based on a trial of continuous choices and based actions so as to alternate forecasts and evaluations often drawn in the practice on past experiences. The plan, nevertheless, would not be effective without an analysis of the data and a control on the real developed activities and on the application of what is being designed.

\begin{tabular}{|c|c|c|c|c|c|}
\hline \multirow[b]{2}{*}{$\begin{array}{l}\text { Sector of economic activity } \\
\text { (ATECO code) }\end{array}$} & \multicolumn{5}{|c|}{ Year event } \\
\hline & 2007 & 2008 & 2009 & 2010 & 2011 \\
\hline A Agribusiness & 5.705 & 5.735 & 5.994 & 6.255 & 5.989 \\
\hline B Fishing & 383 & 394 & 356 & 309 & 282 \\
\hline C Mining & 1595 & 1.439 & 1.270 & 1.222 & 1.091 \\
\hline D Total manufacturing & 211.521 & 195.282 & 151.080 & 148.089 & 136.237 \\
\hline E Electricity, gas, water & 4.483 & 4.180 & 4.171 & 4.044 & 3.740 \\
\hline F Construction & 102.488 & 93.797 & 81.643 & 74.485 & 66.331 \\
\hline G Total trade & 77.022 & 76.010 & 71.190 & 70.338 & 65.551 \\
\hline H Hotels and restaurants & 34.725 & 34.279 & 32.589 & 32.509 & 31.229 \\
\hline 1 Transport and communications & 70.649 & 68.173 & 62.103 & 60.941 & 55.635 \\
\hline J Financial intermediation & 6.948 & 6.854 & 6.950 & 6.986 & 6.442 \\
\hline K Real estate and business services & 53.524 & 53.345 & 51.065 & 51.785 & 49.094 \\
\hline \begin{tabular}{|l}
$\quad$ Pubblic administration \\
\end{tabular} & 25.311 & 23.937 & 25.291 & 24.384 & 22.612 \\
\hline M Learning & 6.095 & 6.088 & 6.453 & 7.655 & 7.889 \\
\hline $\mathrm{N}$ Health and social services & 34.664 & 34.592 & 36.212 & 35.992 & 34.777 \\
\hline 0 Other public services & 33.226 & 34.208 & 36.549 & 39.054 & 37.858 \\
\hline 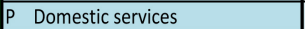 & 2.947 & 3.600 & 3.931 & 4.956 & 4.803 \\
\hline $\mathrm{X}$ Not determined & 154.607 & 148.533 & 128.628 & 124.457 & 118.096 \\
\hline TOTAL & 825.893 & 790.446 & 705.745 & 693.461 & 647.656 \\
\hline
\end{tabular}

Figure 1: The I.N.A.I.L. statistic data

\section{THE SAFETY IN THE CONSTRUCTION SECTOR}

The reference of I.N.A.I.L. data base is important to appraise the evolution with time of the typologies of accident or event that develop in the building sites. In the present research the analysis of the factor has been considered interesting, defined "Ki" or the awareness of the risk from the worker which is considered one of the fundamental elements for the management of the safety in the building site. We believe that the management of the information and the formation of the employees is one of the principal roads to follow for defining a culture of the safety and the prevention in the places of job and particularly in the building sites. The research has wanted to investigate particularly the theme of the management and the control in the building sites proposing the use of a system of sensors, furniture, connected to a "Totem" technological portable that picks up the select data for the monitoring and the control of some hangings held of interest for the correct use of the building site. The data management are set in continuity with the development of a methodology for the project of the yard with the technology BIM realized through 
the definition of families of site elements and the use of the architecture of the phases. A model date-base BIM of the yard that allows updating the data allowing therefore to have sketches always adjourned has been defined. The theme becomes, therefore, the collection of the necessary data. The present job wants to investigate the possibility to recover data and information from a Smart Building Site and to introduce and to manage in the basic date BIM.

The building site is considered, then as a place of extreme dangerousness; the activities that take place expose the workers to serious dangers during the whole work. All over the world the theme of the safety is object of analysis and search of innovative solutions and more proper new systems of management to improve the safety level of the building site. In particular, one of the fundamental aspects is training and information of the operators.

The safety in the building activities should become more than never an automatism, that's why the idea is that systematize automatic or semiautomatic of monitoring can contribute to the definition of a sure and efficient building yard. The development of the electronic technology has allowed a strong reduction of the costs of the elements with a constant diffusion in each sector of active elements for controls of parameters or for actuators of electronic mechanisms; domotics in the new house-building is a typical example. Hence, the trial is to introduce in the yard active elements or active seeds for the monitoring of some physical parameters and the verification of safety distances. For the data management different methodologies are appraised for the harvests and the data management. Several are the variables at the base of the planning of the "system-site" and they are strongly diversified between case and case. The building site is definable as a temporary fine-organization in which a complex structure of fittings, equipments, areas of manoeuvre, stores, offices has the univocal objective to realize the building project.

And it is well known that this organization is strongly correlated with the location, the area and the conditions to the boundary conditions. The Building site resembles for the highlevel of risk for the workers, whit respect to the Italian legislation which is very complex in the definition of the specific risks and in the activity of THEIR evaluation. The tools that according to what anticipated from the law, are to be defined are P.O.S., Plain operational safety, and the P.S.C., plain safety and coordination, that represent the operational manuals of every single building site. These documents are intended to shape and to represent the complex system of variables that determine its oneness and need a punctual planning and management to guarantee a rational and convenient productive trial, further to assure the conditions for the health and the safety of the busy workers.

\section{THE BUILDING SITE DESIGN - SAFETY PARAMETERS.}

The proposed research, is oriented towards the selection, inside the complex system, of variables that characterize the building site of some parameters and some safety distances whose control or monitoring can be useful to the management of the safety of the workers if inserted and if connected to the most complex system of data of the project of the yard with architecture BIM. The study is limited to two themes faced through the system of network, the first one is the control of some environmental parameters connected with the physical risks and the second it is the control of the respect of some safety distances in the site. The search, therefore, wanted to experiment the approach and, eventually, to widen to other variables and other parameters. The select points have been held of particular meaning in relationship to the geometry of the same yard and its location. For what rfers to the physical risks the same they have been valued in relationship to how much anticipated from the safety legislation in theme of microclimate, therefore identified by the monitoring of the temperature, of the speed of the wind and from the rate of damp over that to the noise. Everything is intended to contribute to the maintenance of the conditions of salubriousness and comfort for the workers as prescribed by the norm of prevention accidents. The control of the safety distances is of fundamental importance in the general management of the system-building site: the rules and the laws about accident prevention, in fact, are based on the imposition of distances and limits to the half and the personnel. The idea that is developed, therefore, is the creation of a smart grid in the passive site, fixed in building site that returns a series of information regarding parameters physical environmental and to a site smart map that individualizes a system of areas and zones of danger and invitations to the use of the correct viability of yard both vehicles and workers.

The proposal is based on the real time collection of data regarding the physical and environmental parameters and of the distances that picked up meets in a system of control of the based yard on the architecture BIM. It is common idea that the insertion of the yard in the environment meaningfully conditions its organization, in fact, is in demand to the planner of the yard, besides, to minimize the interferences checking the interactions with the outside through elements what the enclosure and the accesses, over that the position of the site in comparison to the external viability and therefore also the general environmental condition defined by the nature of the ground by the climatic conditions from the availability of technological nets. For the carrying out of the search we have followed what anticipated from the UNI STANDARDS $10756 / 98$ and particularly its elaborate graphic descriptive of a general plan of building site for a generic building intervention of new building type conventional, in an optimal considered situation. 


\begin{tabular}{|c|c|c|c|c|c|}
\hline & Bluetooth & Wifi & UWB & RFID & ZigBee \\
\hline Standard & IEEE 802.15.1 & IEEE 802,11b/g & $\begin{array}{c}\text { IEEEE } \\
802.15 .3\end{array}$ & \begin{tabular}{|c|} 
ISO/EPC \\
Global
\end{tabular} & \begin{tabular}{|c|c} 
IEEEE \\
802.16 .4
\end{tabular} \\
\hline Big Rate & $2.1 \mathrm{Mbit} / \mathrm{s}(\mathrm{v} .2 .0)$ & $11 / 54 \mathrm{Mbit} / \mathrm{s}$ & $480 \mathrm{Mbit} / \mathrm{s}$ & $\begin{array}{l}10-50 \\
\mathrm{kbit} / \mathrm{s}\end{array}$ & $250 \mathrm{kbit} / \mathrm{s}$ \\
\hline Range (per single hop) & $<10 \mathrm{~m}$ & $<100 m$ & $<40 \mathrm{~m}$ & \begin{tabular}{|l}
$<1 \mathrm{~m}$ pass. \\
$-80 \mathrm{~m}$ att.
\end{tabular} & \begin{tabular}{|c|c|}
$<80 \mathrm{~m}$ \\
$(\mathrm{Km}$ per \\
$\mathrm{m}-\mathrm{hcp})$ \\
\end{tabular} \\
\hline cost & Medium & High & Medium & Low & Low \\
\hline Security Level & Low & Medium & Medium & Medium & High \\
\hline Network Topology & Star Only & Star typically & 1 & $\begin{array}{l}\text { Star }-> \\
\text { Reader } \\
\end{array}$ & $\begin{array}{l}\text { Star, Cluster } \\
\text { tree, Mesh }\end{array}$ \\
\hline Energy Consuption & Medium & High & Medium & Low & Very low \\
\hline
\end{tabular}

Figure2. The different standard of the data transmission

The strong reduction of the costs of the electronic components makes easier and easier the availability of small intelligent elements of easy use. The experimentation of automatic systems in the building sector has already been departing FROM 80'S in Japan. To the base we have put the principle of base to translate the logical CIMs (Computer Integrated Manufacturing) of the industry, in logical CIC (Computer Integrated Construction) in the sector of the constructions. Solutions of automation of phases of workmanship are also experimented in the yard, to improve its productivity with times and costs reduction. To actively check the yard different technologies and methodologies are being experimented. In general, to the goals of the search we believe that the monitoring with the collection of the data in real time on the conditions of the yard and the use of the spaces becomes essential to identify alarms or conditions of danger. Different studies and applications described in literature set as solution the endowment to the workers of active equipments or active seeds that for instance converse with data loggers of receipt of the data for the verification of the safety endowments based on technologies GPS and RFID, essentially formed by a tag (active or passive) and a reader, the tag contains a microchip and a spar, which can be passive (without battery) or active (with battery); while the reader, receives the data that can be transferred to a computer. Comparison they are set to different technologies of transmission of the data synthesis in chart: they are mails to comparison in the following chart. There are many available studies on the matter and with satisfactory levels of precision, in all the cases two are the aspects that must characterize the employment in a building yard; in first place, the infrastructure must be flexible, to avoid a notable waste of resources in terms of time and money, in all the cases in which, the intention is to implement it or to modify; in according to place the ability of data transmission must guarantee a least level of communication in every point of the orchestrated space. Necessity to have infrastructures preinstalled is certainly a limit since it asks for preliminary and autonomous operations that doesn't very often find a full sharing. It is necessary, nevertheless, to establish that preliminary works of setup and organization of the yard are necessary and not eliminable. It is tried to involve in the organization of the system of monitoring proposed of the invariant ones of yard or areas and zones that stay stable during the whole run of construction,

And it is to be considered, also, that all the elements must be proper for the positioning to the outside. Basing the system of monitoring on the endowment from the workers of devices or equipments determines that the whole system can negatively be influenced by a missed respect of the procedures of the same employees. For these motivations it is necessary to assess additionally, passive systems that totally bring information and independent indications from the workers themselves.

\section{THE RESEARCH PROPOSAL}

The proposal of the present job is therefore passive type and based on the positioning of elements able to collect and monitor the parameters identified over whether to set in safety the vehicular and pedestrian viability. The principal element of the system is a technological Totem self powered that has the assignment of monitoring the levels of temperature, speed of the wind and the noise over whether to pick up the signal coming from the elements set for the control of the viability. Elements are also self powered, whit the assignment to send signals of alarm when points of conflict are verified among the two viabilities. Some elements are destined to the control of the operational areas of the means as projected. In synthesis a tied up passive system to the planning of the site of having yard the assignment proposes him to check some hangings and to transmit them to the central system of elaboration. The communication happens on net LAN (Local Area Network) based wireless on the specific IEEEs $802.11 \mathrm{~b}$.

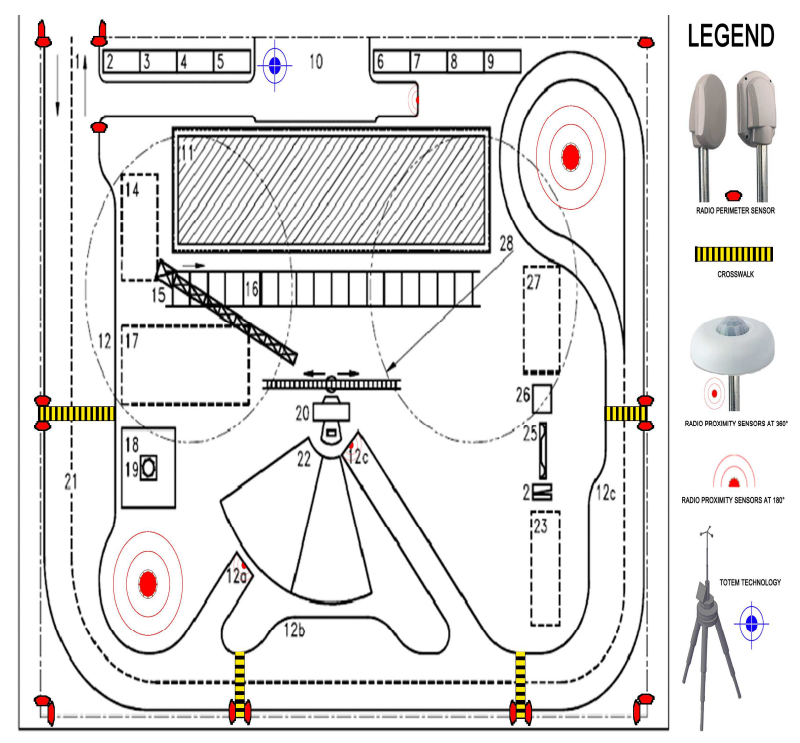

Figure3. The building site map with sensor based of UNI 10756. 
The job exposes a proposal that is in progress of improvement and it will be object of activity of experimentation. At the base of the search the are some fundamental concepts. In first place the network must be integrated in the most complex activity of planning of the yard and therefore the same both one of the variables that define the Lay out; the second aspect is that of the versatility of the system that allows to suit for different configurations of yard. In the preliminary evaluations the attention is turned, in this first phase, to some environmental parameters and some dimensional parameters which can be summarized in an activity of environmental monitoring and the noise besides the control of the dimensional respect of some sensitive areas of the system. The system sends the data to the totem from the satellite elements that are connected with a computer with software BIM.

A fundamental fact that the system BIM is in a position to read and to represent is the parameter time. This feature, that agrees to have dynamic displays of the only one project, agreed, to be able to simulate all the phases of building site. The simulation of all operating phases so agreed to do dynamic consideration on all the appearance of the site: quantity of the supplies, definitions of the different areas of storage, location of the means, makings orders, and, in this case the evaluations of safety.

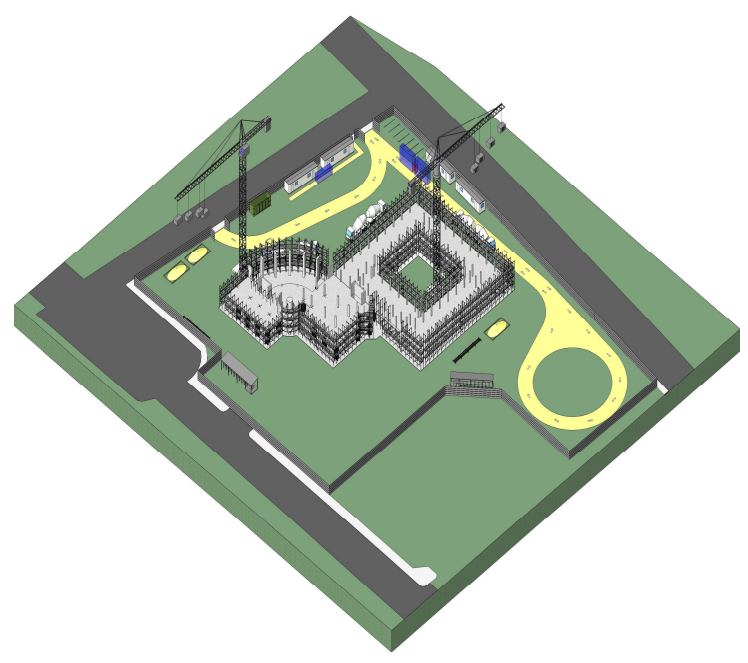

Figure4. The representation of the warning data in to BIM data base.

Through the software BIM the project in $3 \mathrm{D}$ is available both of the building that of the building site, from which a site map can be derived as reference with the location of the sensors. The sensors transmit, when a trespass happens, a signal of united warning to the position in the site map of the point in which the message is launched bringing over of it that the coordinates the schedule of the recording and eventually an in partnership code to the type of alarm. This site map, connected in initial phase to the project, is adjourned as soon as automatically the sensors they bring the trespasses and therefore they implement the in partnership file. in this way the result is a file of points adjourned during the jobs with location, schedule and type of message.

\section{CONCLUSIONS}

In conclusion the research has investigated the possibility to realize a passive net of management and control in the building site connected to the BIM data base. some characteristic flexibilities and facility of installation have been asked to the system; automatic monitoring; reductionof costs of management and maintenance; resistance to the atmospheric conditions and of yard; reliability of the outputs; raised energetic autonomy. The job in initial phase has returned some interesting results that push to the second phase for which have been anticipated further experimental activities. We believes that the technological development of the building site is the correct direction for a sure and efficient site.

\section{REFERENCES}

[1] De Luca Picione, M. and Mottola, V - Il progetto dinamico del cantiere edile, Il Sole 24 ore, Milano 2009.

[2] Mecca, S.- Comprendere il cantiere, Edizioni ETS, Pisa 2002

[3] Norma UNI 10756/98 Building - Executive design Definitions, organization and contents of executive plans for new constructions

[4] Kiviniemi M., Sulankivi, K., Kähkönen, K., Mäkelä T. and Merivirta M. L. - BIM - BASED Saftey Management and Communication for Building Consruction VTT Research Notes 2597 
